Abstract PS3:58 Table 1 Mean values of lipid profile at time 1 and 2 (result units in $\mathrm{mg} / \mathrm{dl}$ )

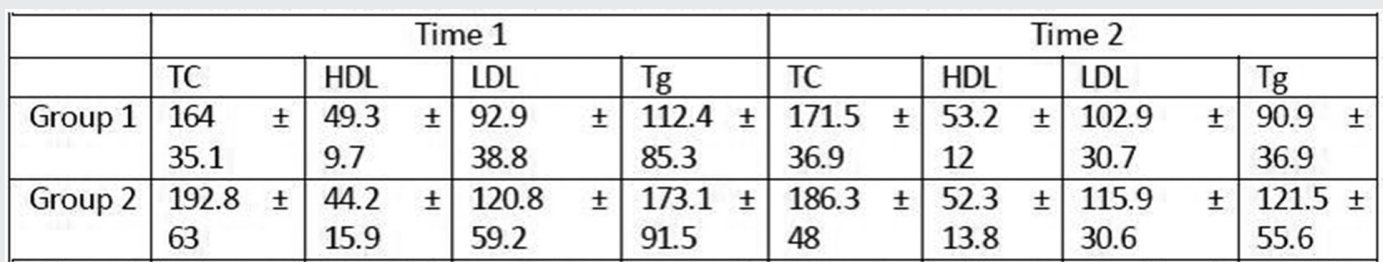

TC: total cholesterol; HDL: high densitylipoprotein; LDL: low-density lipoprotein; Tg: triglycerides

between proteinuria and LDL levels $(r=0.67, p=0.017$ and $\mathrm{r}=0.74, \mathrm{p}=0.009$ respectively).

Conclusion Our study shows that Juvenile SLE patients with LN tend to have more abnormalities of lipid profile than patients without LN, namely with higher TC, LDL and Tg, and lower HDL.

A significant positive correlation was found at time 2 between proteinuria and TC and LDL levels, reflecting that the severity of proteinuria correlates with abnormalities in lipid profile.

These results reinforce that juvenile SLE population, namely with $\mathrm{LN}$, should have their CV risk factors, such as lipid profile, carefully monitored.

\section{PS3:59 IS THERE A DIFFERENCE BETWEEN LIPID PROFILE BEFORE AND AFTER LUPUS NEPHRITIS TREATMENT IN PATIENTS WITH JUVENILE SYSTEMIC LUPUS ERYTHEMATOSUS?}

II Santos Jorge, ${ }^{2} \mathrm{M}$ Guerra, ${ }^{3} \mathrm{~A}$ Águeda, ${ }^{4,5} \mathrm{R}$ Ferreira, 5,6 M Rodrigues, ${ }^{5,6}$ | Brito. ${ }^{1}$ Physical Medicine and Rehabilitation Department, Centro Hospitalar do Porto, Portugal; ${ }^{2}$ Rheumatology Department, Centro Hospitalar Vila Nova de Gaia/Espinho, Vila Nova de Gaia, Portugal; ${ }^{3}$ Rheumatology Department, Centro Hospitalar do Baixo Vouga, Aveiro, Portugal; ${ }^{4}$ Rheumatology Department, Centro Hospitalar de São João, Porto, Portugal; ${ }^{5}$ Faculty of Medicine of Porto University, Porto, Portugal; ${ }^{6}$ Paediatric Rheumatology Unit, Centro Hospitalar de São João, Porto, Portugal

\subsection{6/lupus-2018-abstract.106}

Purpose Renal involvement is one of the major determinants of outcome in patients with Systemic Lupus Erythematosus (SLE). Dyslipidaemia occurs frequently in Juvenile SLE. This study aimed to determine if there were differences in lipid profile in Juvenile SLE patients with lupus nephritis (LN) in two time points, time 1 at $\mathrm{LN}$ diagnosis and time 2 after 612 months of treatment.

Methods Retrospective analysis of the lipid profile of a population of Juvenile SLE patients with $\mathrm{LN}$ in two time points. Lipid profile evaluation included total cholesterol (TC), triglycerides (TG), high density lipoprotein (HDL) and low-density lipoprotein (LDL). Statistical analysis was performed using $\mathrm{T}$ Student Test. Significance level was set as $<0.05$.

Results At LN diagnosis, time 1, the mean TC was $198,1 \mathrm{mg} /$ dL ( $\pm 62,9)$, TG was $175,1 \mathrm{mg} / \mathrm{dL}( \pm 89,5)$, HDL was $44,3 \mathrm{mg} /$ $\mathrm{dL}( \pm 16,9)$ and $\mathrm{LDL}$ was $120,6 \mathrm{mg}( \pm 61,2)$. At time 2 , the mean TC was $188,2 \mathrm{mg} / \mathrm{dL}( \pm 47.0)$, TG was $126,3 \mathrm{mg} / \mathrm{dL}$ $( \pm 57.1)$, HDL was $52,3 \mathrm{mg} / \mathrm{dL}( \pm 13,8)$ and LDL was $115,9 \mathrm{mg} / \mathrm{dL}( \pm 30,6)$.$) . Despite higher levels of TC, TG and$ LDL levels at time one, the variation of lipid profile between the two time points did not reach statistical significance.
Conclusions Our study showed that the lipid profile of LN patients tends to improve with LN treatment, as showed by the lower levels of TC, TG and LDL and higher levels of HDL observed at time 2 .

\section{PS3:60 INCIDENCE OF VASCULITIS IN HOSPITALISED LUPUS PATIENTS}

${ }^{1} \mathrm{Z}$ Mirfeizi, ${ }^{2} \mathrm{ZH}$ Shariati Sarabi, ${ }^{3} \mathrm{E}$ Atabati, ${ }^{4} \mathrm{R}$ Ranjbar, ${ }^{1} \mathrm{M}$ Sahebari, ${ }^{5} \mathrm{~K}$ Hashemzadeh. ${ }^{1}$ Associate Professor of Rheumatology, Faculty of Medicine, Mashhad University of Medical Sciences, Mashhad, Iran; ${ }^{2}$ Professor of Rheumatology, Faculty of Medicine, Mashhad University of Medical Sciences, Mashhad, Iran; ${ }^{3}$ Assistant Professor of Rheumatology, Faculty of Medicine, Birjand University of Medical sciences, Birjand, Iran; ${ }^{4}$ Emergency Physician Specialist, Faculty of Medicine, Mashhad University of Medical Sciences, Mashhad, Iran; ${ }^{5}$ Assistant Professor of Rheumatology, Faculty of Medicine, Mashhad University of Medical Sciences, Mashhad, Iran

\subsection{6/lupus-2018-abstract. 107}

Introduction Systematic Lupus Erythematosus (SLE) is an autoimmune disease which is associated with multiple target organ damage. Multiple hospitalizations can occur during their lifelong. Systemic vasculitis manifestation is one of the most important reasons leading to refer to rheumatology clinics. The aim of this study is to evaluate different picture of systemic vasculitis in lupus patients.

Materials and methods Eighty-one known SLE patients based on 2012 Revised Criteria who were visited in Rheumatology Department of Imam Reza Hospital from January 2012 to December 2014 having undergone a thorough physical examination by rheumatologist, classified based on demographic and SLE-related vasculitis manifestations. We focused on vasculitis manifestation in this group of patient.

Results In total $17.3 \%$ of our lupus patients were diagnosed as vasculitis. In the vasculitis group cutaneous vasculitis (50\%), thromoembolic events $(7 / 1 \%)$, cerebral vasculitis $(7 / 1 \%)$, retinal vasculitis $(7 / 1 \%)$ and aortic involvement were detected. Systemic vasculitis is the second most common causes for referring to the hospital. Mean SLEDAI and SLICC scores were $22.79( \pm 13.29)$ and $1.95( \pm 1.46)$, in vasculitis group respectively.

Considering SLICC SLE Criteria, prevalence of vasculitis lesion were found more often in ocular, renal, gastrointestinal and CNS system $(\mathrm{p}=0.03,0.01,<0.001,<0.03$ respectively.

Conclusion Vasculitis manifestation in lupus probably is one of the most important and life threatening complication which may have brought these patients to rheumatology centre. In this study, we found that vasculitis may be the first presentation of SLE patients with severe organ damage with no previous history of this disease. 